\title{
Propagation measurements and analysis on MF and HF bands in urban areas in The Netherlands
}

\author{
Koos (T. W. H.) Fockens ${ }^{1}$, Robert Vogt-Ardatjew² ${ }^{2}$ Frank Leferink ${ }^{3}$, Fellow, IEEE
}

\begin{abstract}
Using a new propagation measurement set-up, which produces a high number of data, enabling a proper statistical analysis, and resulting in very concise results, propagation measurements were performed and analized at 16 residential locations in The Netherlands in the frequency range from 1.8 to $28 \mathrm{MHz}$. In the whole frequency range the propagation loss appear to be higher than according the International Telecommunication Union ground-wave propagation model might be expected. Also typical characteristics of that model are not present, but instead the propagation shows a constant roll-off in dBs per decade, which slope is increasing with frequency. A regression curve could be established, and constants filled in. This statistical information may be used for building an accumulation model to lay a causality between source powers, source densities, and local Man-Made Noise levels.
\end{abstract}

Index Terms-Accumulation of man-made radio noise, EMC, Propagation.

\section{INTRODUCTION}

In a study on the accumulation of man-made radio noise (MMN) by large numbers of noise sources, the propagation of the EM waves from individual noise sources to a point of aggregation is very relevant. MMN in the frequency range of 0.47 to $50 \mathrm{MHz}$ was investigated earlier [1]. A further statistical study of the measurement results revealed that there is a strong correlation between the measured noise floor and the density of habitation [2]. These results raise the question of how does the radio noise propagate in these populated areas, and what is the propagation loss with distance. In the earlier mentioned frequency range, the wavelength vary from 6 to $600 \mathrm{~m}$. As the field strength measurements were done at an antenna height of 2 meters, and the sources are located in homes mostly at the first, second or third floor, or in more dense areas in higher apartment buildings, these heights are also relative low. So, a very reasonable presumption is that the propagation may be described by the theory of the groundwave $(\mathrm{GW})$ propagation.

For a well understanding of this theory we will look into a part of the history. After Marconi had proven the ability of long range radio communication by his transatlantic experiments in 1901 the need for a theoretical explanation was first fulfilled by Zenneck [3] and Sommerfeld [4]. Zenneck introduced a surface wave as a solution for the Maxwell equations. Sommerfeld analyzed the case of a vertical Hertzian dipole over a lossy ground, and came also to the surface wave as described by Zenneck, hereafter called "Zenneck Surface Wave", ZSW. The main property of this ZSW is that the energy is trapped on the surface, and that the roll-off with distance is $10 \mathrm{~dB} /$ decade, instead of $20 \mathrm{~dB} /$ decade for a wave in free space. From 1936 on Norton showed in [5], [6], [7] that the ZSW could only exist under extreme conditions, and that Sommerfeld had made a mistake in his theory by a faulty interpretation of the square root of a complex variable, this resulting in the so-called "sign controversy". A complete overview of this history can be found in [8] and in [9].

In [10] Norton defined the GW as "A Radio Wave that is propagated through space and is, ordinarily, affected by the presence of the ground", with excluding any other reflections than against ground, as for example the ionosphere. In his solution the field strength at a distance from a transmitting antenna, a vertical dipole or a magnetic loop with the winding in a vertical plane, is given by an equation in [10], showing a sum of three terms. The three terms are corresponding respectively to the direct wave, the ground reflected wave, and the surface wave, generally called the "Norton Surface Wave", NSW. In the derivation by Norton the NSW is mathematically the result of the subtraction of the optically reflected Reflected Wave from the total of wave energy that is interacting with the ground. When the transmitting and the receiving antenna are close to the ground with respect to the wavelength the direct and the reflected wave will cancel, so the NSW is left. It is important to realize that the NSW is not trapped on the surface, in contrast to the $\mathrm{ZSW}$, and that the roll-off with distance is minimal $20 \mathrm{~dB} /$ decade of distance. The values of the attenuation can be looked up on graphics in [11], a description is given in [12], and may be calculated by means of [13].

In this study, propagation in urban areas, all three wave paths in the GW theory are involved. The distance varies from 50 to $1000 \mathrm{~m}$, in the measurements the transmitter antenna is at a level of $3 \mathrm{~m}$ and the receiving antenna at $2 \mathrm{~m}$. That means that next to the NSW the direct and reflected wave may play a role, especially for the short distances and for the upper part of the frequency range. For all three paths the buildings may damp, reflect, or scatter all three waves. Especially tall vertical constructions as street lamps, towers, etc. may cause scattering, which, depending on conductivity and the ratio between height and wavelength, may cause forward, back, and random scattering. Buried conductors in the earth, like all kinds of cables and metal pipes, may enhance the ground conductivity, so decrease the attenuation of the surface wave.

1 Koos (T.W.H.) Fockens is with the University of Twente, Enschede 7522NB, The Netherlands, and member of the EMC/EMF committee of the Dutch radio amateur society (VERON), Arnhem $6801 \mathrm{BD}$, The Netherlands.

2 Robert Vogt-Ardatjew is with the University of Twente, Enschede 7522NB, The Netherlands,

3 Frank Leferink is with the University of Twente, Enschede 7522NB, The Netherlands, and also with Thales Netherlands, Hengelo 7554RR, The Netherlands. 
This is especially the case at the lower frequencies with the greater skin depth. We must remark here that overhead cabling is not used in The Netherlands, so does not play a role in our measurements.

In the last decades propagation studies, theoretically and empirical, [14], [15], are mostly concentrated on VHF and UHF frequencies, especially in urban areas. In this frequency range the wavelength is small to very small with relation to the dimensions of the objects in that environment. For the MF and $\mathrm{HF}$ range the wavelengths are larger or equal to these dimensions. This also means that in the VHF/UHF range the wave interaction with the ground is less relevant and that free space, reflected, and scattered paths dominates. As a consequence these studies are not relevant in our case.

There is a very limited number of studies about propagation in urban areas on frequencies below $30 \mathrm{MHz}$, and they are in essence limited to Medium Wave broadcasting signals. In [16] an extensive measurement campaign and theory building has been performed about the propagation from a local MW transmitter site North of London, through the city centre to the South of London, with a total range of $60 \mathrm{~km}$. Three frequencies were involved, and interesting phenomena were recorded and theoretically explained. Herein the built-up environment with its buildings was modelled as a "bed of nails" like structure. However, in our study the relevant frequency range is higher, and the distance much shorter. In [17] field strength measurements were perform on the propagation of a local MW transmitter in São Paulo, Brazil. One of the results that were reported is: "the prediction model of Rec. ITU-R P.368-9 overestimates the measured field during daytime". Consequently the GW propagation losses were higher than expected. In [18] propagation measurements on HF frequencies were reported in open desert areas, as well as in urban environments. The conclusion in this report were that the losses in open areas matched the expectation within a few $\mathrm{dB}$, but in urban areas a diversity of propagation losses was found when measured the GW propagation path, and varied all over the city. A need was concluded to develop a prediction model for urban environments. Reference [19] reports a new MF and HF GW propagation model for urban areas. It includes a fundamental addition to the Norton model by building-complex parameters and height-gain factors. The model applies for areas with high and tall buildings in particular. Only validating measurements at the medium wave frequencies were reported.

We conclude that the existing models for propagation below $30 \mathrm{MHz}$ handle urban areas as a local disruption in a wider area, mainly for the purpose of coverage studies of broadcasting transmitters. For our application, being short range intra-urban propagation of man-made noise in the MF and $\mathrm{HF}$ range, there is no sufficient scientific information available about measurement data, nor theoretical models, useful for a statistical modeling of accumulation of spatial spreaded noise sources, so leading to a conclusion that a measurement campaign was necessarily.

The paper is organized as follows. In section II we describe the measurement methods, in section III the data processing and some theoretical aspects. Section IV describes the statistical analysis and the results thereof. Our conclusions are summarized in section V. In Appendix 1 we describe validation measurements, and in Appendix 2 we calculate the field strength above Perfect Electrical Conducting ground. At the end, Appendix 3 derives the equations to make field strength measurement corrections in the near field.

\section{DESCRIPTION OF THE MEASUREMENTS}

The goal of the measurement campaign is to gather statistical data about transmission loss as a function of distance, frequency, and of density of habitation. Thereto we need measurements on several locations in diverse environments. From the list of measurement locations, earlier used in [1], we selected a number of 16 , and enumerates them in Table I.

TABLE I. Overview of measurement locations.

\begin{tabular}{|c|c|c|c|c|c|c|c|c|c|c|c|}
\hline \multicolumn{2}{|r|}{ Location } & & \multicolumn{7}{|c|}{ Number of homes in radii of the annuluses } & \multicolumn{2}{|c|}{ Environment } \\
\hline No. & Call sign & City & $0-50$ & $50-100$ & $100-200$ & $200-300$ & $300-400$ & $400-500$ & Total & Type & Group \\
\hline 1 & PAORLM & Driebergen & 6 & 2 & 48 & 108 & 258 & 249 & 671 & Rural & 1 \\
\hline 2 & PA3AWN & De Heurne & 3 & 10 & 11 & 35 & 26 & 23 & 108 & Res. 1 & \\
\hline 3 & PAORYL & Huis ter Heide & 5 & 15 & 98 & 147 & 54 & 49 & 368 & Res. 1 & \\
\hline 4 & PAOJMG & Drachten & 7 & 7 & 66 & 110 & 107 & 130 & 427 & Res. 1 & \\
\hline 5 & PAOWTA & Apeldoorn & 6 & 14 & 62 & 225 & 261 & 225 & 793 & Res. 1 & \\
\hline 6 & PAOHTT & Ommen & 12 & 54 & 153 & 176 & 144 & 112 & 651 & Res. 2 & II \\
\hline 7 & PC7M & Goor & 23 & 43 & 158 & 133 & 158 & 193 & 708 & Res. 2 & \\
\hline 8 & $\begin{array}{l}\text { PAOVBR } \\
\end{array}$ & Nijmegen & 27 & 71 & 160 & 184 & 338 & 379 & 1159 & Res. 2 & \\
\hline 9 & PCOWP & Hengelo(Ov) & 11 & 39 & 108 & 358 & 313 & 423 & 1252 & Res. 2 & \\
\hline 10 & $\begin{array}{l}\text { PA1AT } \\
\end{array}$ & Assen & 24 & 63 & 152 & 382 & 406 & 308 & 1335 & Res. 2 & \\
\hline 11 & \begin{tabular}{|l|} 
PBOAIR \\
\end{tabular} & Hengelo(Ov) & 31 & 64 & 366 & 363 & 447 & 626 & 1897 & Res. 2 & \\
\hline 12 & PAOWJG & Nieuwegein & 22 & 55 & 348 & 540 & 446 & 518 & 1929 & Res. 2 & \\
\hline 13 & PAORSM & Amersfoort & 30 & 86 & 210 & 407 & 323 & 452 & 1508 & Res. 3 & III \\
\hline 14 & PA3BME & Driebergen & 21 & 94 & 274 & 446 & 407 & 441 & 1683 & Res. 3 & \\
\hline 15 & PA3GXD & Meppel & 52 & 106 & 369 & 511 & 533 & 575 & 2146 & City & \\
\hline 16 & PH1E & Eindhoven & 34 & 57 & 274 & 448 & 656 & 715 & 2184 & City & \\
\hline
\end{tabular}

They all belong to contributing radio amateurs, the locations are coded in the table and further on in accordance with their radio call signs. Also the city is given and numbers of homes around that locations. The type of environment is given as defined in [1]. According these environments three groups of locations, I, II and III, are created with increasing density of habitation.

The principle of the measurements is based on placing a low power beacon transmitter at a fixed location and drive with a mobile field strength (FS) measurement system in a fixed traject from that location away up to a distance of about 1 kilometer and back via different path, depending on the local topography, and selectively measuring the field strength generated by that beacon transmitter. This drive is repeated for all test frequencies over the same traject: 1.85 (160 m band), 3.57 ( $80 \mathrm{~m}$ band), 7.07 (40 $\mathrm{m}$ band), 14.07 (20 $\mathrm{m}$ band), 21.07 (15 $\mathrm{m}$ band), and 28.07 $\mathrm{MHz}$ (10 $\mathrm{m}$ band). During the drive repeatedly measurement samples are taken at random varying distances 8 - 16 meters between themselves, resulting in a high number of samples.

The special designed beacon transmitter consists of a 19 inch cabinet, a $4 \mathrm{~m}$ high rod antenna, and two metal plates forming a counterpoise grounding, see Photo 1 . Next to the transmitter, a commercial Elad FDM-DUO SDR transceiver, the cabinet contains an antenna matching circuit and a Morse code ID generator, which modulates the transmitter carrier in Frequency Shift Keying with a shift of $100 \mathrm{~Hz}$. That is well within the bandwidth of the measurement receiver, so that the measurement is not disturbed by the ID transmission. The transceiver output power is set at 1 Watt. The RF carrier is measured at the measurement receiver. In principle this includes noise within the receiver bandwidth, but during the measurements the level of the beacon signal is that strong that external and internal noise does not contribute to the measurement result significantly. 


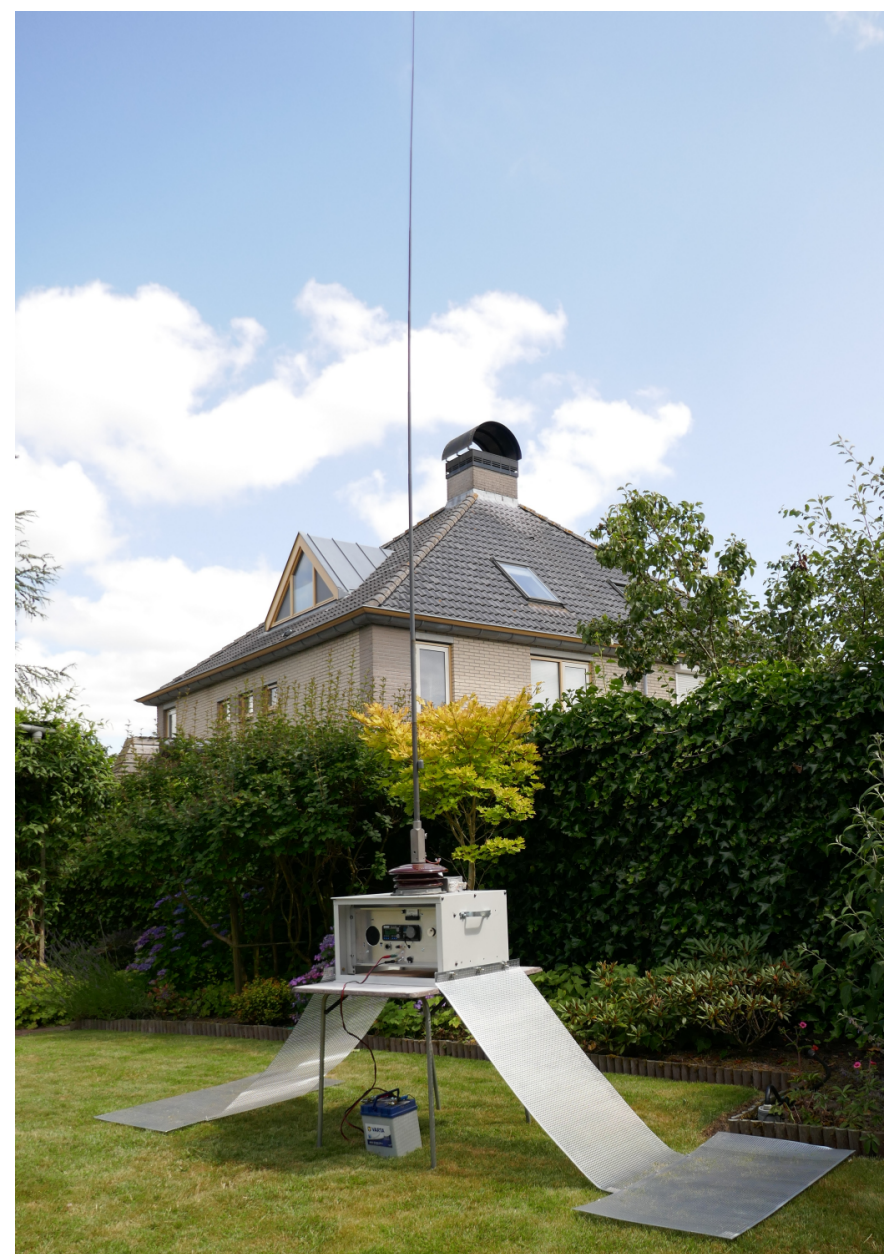

Photo 1. View of the beacon transmitter.

For calibration the field strength has been measured at a distance of $25 \mathrm{~m}$ on a flat and undisturbed piece of grass land by using a calibrated magnetic antenna, the Rohde \& Schwarz Z2 loop antenna. For the near field (lower frequencies) corrections were made according correction factors, derived in Appendix 3. Also for ground losses (upper frequencies, very small) compensations, calculated with GRWAVE, [13], has been calculated and applied. From these measurements the actual effective isotropic radiated power (eirp) has been calculated for each test frequency. The eirp values are to be used as calibration numbers in the postprocessing of the field strength measurements.

The mobile FS measurement system was build in a passenger car. On the roof an active E-field antenna system is mounted, see Photo 2. The antenna groundplane as shown is well grounded on the roof and the bodywork of the car by a low impedance capacitive coupling through two self-adhesive copper strips on the roof, behind the luggage carrier strips. The capacitance per strip is $10 \mathrm{nF}$. Fig. 1 shows the principle diagram of the measurement system, and Photo 3 the equipment inside the car.

The calibration of the mobile FS measurement system has been carried out for the system as a whole, including the car. The reference antenna method has been used, using the same Rohde \& Schwarz EZ2 loop antenna as a reference as also used for calibration the transmitter. A beacon transmitter at a distance of 40 meters produced a vertical polarized test signal.

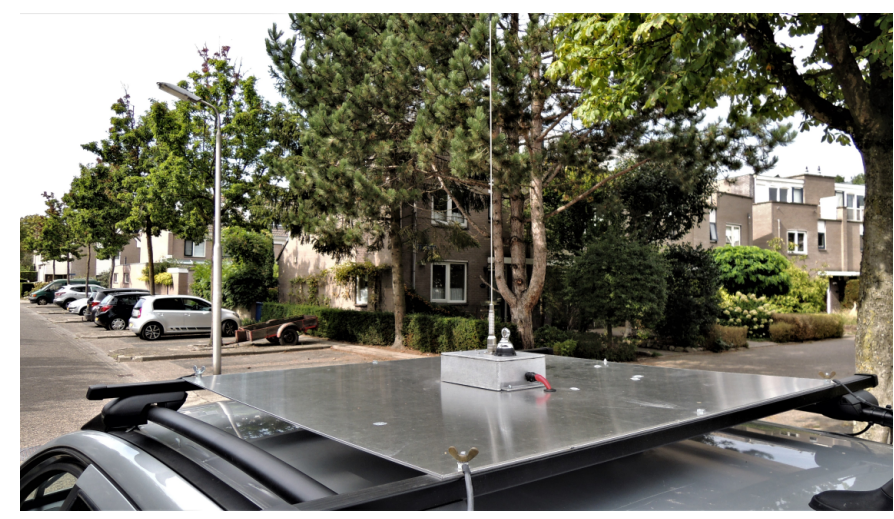

Photo 2. Measurement antenna system.

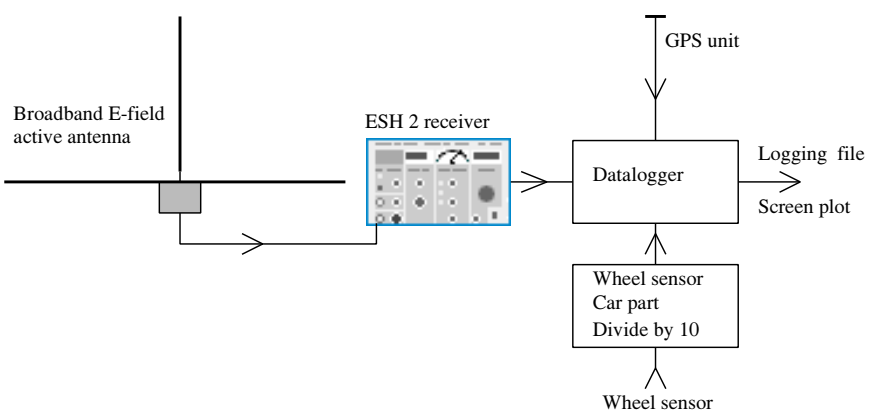

Fig.1. Set-up of the mobile field strength measurement system.

The reference antenna was positioned at the same height as the E-field antenna on the car. For the frequencies, where the Eand $\mathrm{H}$-field divers because of the effect of the near field, a compensation has been made, see Appendix 3. Also the directivity of the mobile set-up has been measured at 5 directions from $0^{\circ}$ (front) to $180^{\circ}$ (back of the car). For all test frequencies a small difference in antenna factor was observed, maximal sensitivity for a signal arriving at the front of the car, and a minimum in the sensitivity for signals arriving from the back side of the car.

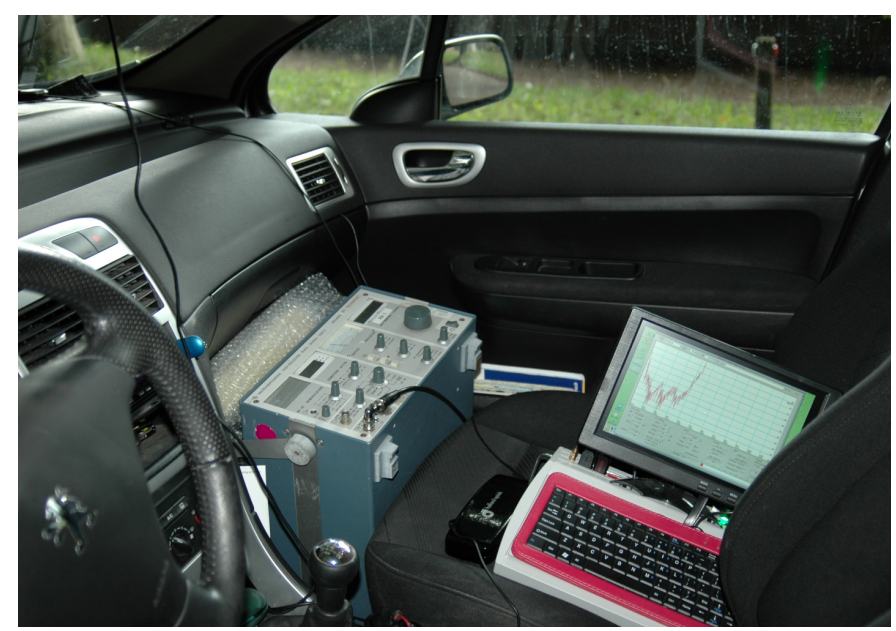

Photo 3. Measurement equipment.

The amplitude of this directional variation is lowest at 1.8 MHz: $0.3 \mathrm{~dB}$, and highest at $25 \mathrm{MHz}$ : $1.3 \mathrm{~dB}$. For each test frequency a cosine function approximation has been derived. An algorithm has been developed to estimate the direction of 
arrival of the beacon signal during the measurement drives from the GPS data, using the stored location of the beacon, so to compensate for directional variations in the antenna-factor in real time.

For validating the measurement system and method measurement drives has been performed in flat and open areas as described in Appendix 1. Concerning seasonal effects on the propagation loss, monthly measurements were carried out throughout a full year, from September 2019 to November 2020 , on a fixed traject, partly in populated area, and partly over open land. A clear relation with seasonal variation in soil humidity was found, but the maximal deviation from the median on the loss measurement, as caused by a total of $10 \%$ to $90 \%$ spread, was very limited, and varied from $1(1.8$ $\mathrm{MHz})$ up to $3 \mathrm{~dB}(28 \mathrm{MHz})$. As a result no corrections were applied in our urban measurements.

\section{DATA PROCESSING}

\section{A. Decimation of samples and the statistical approach thereof}

For the purpose of the accumulation study an estimator is required in this experiment that is representing the propagation loss. We take here the expected value from the probability distribution function (pdf) of the propagation loss, thus the mean value $\mu$ in the pdf.

But samples of FS levels are actually measured, which levels are normalized for an effective isotropic radiated power of 1 Watt, transmitted by the beacon transmitter. These FS levels are used as an intermediate result, they can be used to compare with the FS levels that are resulting from calculations according the ITU GW propagation model.

In the post processing the measurement samples are grouped into bins, representing ranges of distances. The samples, inputted in those bins, can be measured on a single location, on a group of locations, or on all locations. They may be combined during the post processing in those bins. In this way a kind of parallel processing is achieved from: 1st the individual locations, 2nd the three groups of locations with comparable density of habitation, and 3rd from all measurement locations totalized together.

From each bin an average value $A v$ and a median value $M e$ from $N$ samples can be calculated. Generally, $A v$ appear to equal $\mu$ nearly in the measurement results. For higher numbers of $N A v$ and $M e$ approach the pdf mean, and so the mean value, $\mu$. In this case we use the linear values of the field strength $e$ for the statistical calculation, and the results are converted back in logarithmic values $E[\mathrm{~dB} \mu \mathrm{V} / \mathrm{m}]$. In here the statistical processing complicates by the fact that in this field of engineering, basically, FS values are measured in a linear quantity, $\mu \mathrm{V} / \mathrm{m}$, on which the statistics apply, but the further arithmetics and presentations are done in logarithmic quantities, e.g. in $\mathrm{dB} \mu \mathrm{V} / \mathrm{m}$ (FS) or $\mathrm{dB}$ (propagation loss). For the average and median values this is not a problem as they can be converted from linear quantities into $\mathrm{dBs}$ and vice versa unambiguously. But deviations translate from linear into logarithmic values for a negative and for a positive deviation differently. This makes it necessarily to define and describe the complete processing procedure.

Each bin $j$ delivers an average value $e_{A v, j}, E_{A v, j}=20 * \log$ $e_{A v, j}$, and an estimated Standard Error $e_{S E, j}$. The error will be split up in the logarithmic quantities $E_{S E+, j}$ and $E_{S E-, j}$ :

$$
\begin{aligned}
& E_{S E+, j}=20 * \log \left(\frac{e_{A v, j}+e_{S E, j}}{e_{A v, j}}\right) \\
& E_{S E-, j}=20 * \log \left(\frac{e_{A v, j}-e_{S E, j}}{e_{A v, j}}\right)
\end{aligned}
$$

Herein is $E_{S E+, j} \geqslant 0, E_{S E-j} \leqslant 0$, and because of the asymmetry in the ratios $\left|E_{S E-, j}\right| \geqslant E_{S E+, j}$. The median FS value $E_{M e, j}$ has to be normalized for an effective radiated power from the beacon transmitter of 1 Watt:

$$
E_{N, M e, j}=E_{M e, j}-P_{t x}[\mathrm{dBW}]
$$

To calculate the propagation loss $L$ the FS median is subtracted from the FS that would be measured when the ground is Perfect Electrical Conducting, PEC:

$$
\begin{aligned}
L_{M e, j} & =E_{P E C, j}-E_{N, M e, j}[\mathrm{~dB}] \\
l_{M e, j} & =10^{\left(L_{M e, j} / 20\right)}
\end{aligned}
$$

Because of the sign in (4) the standard errors in $L$ will be reversed:

$$
\begin{gathered}
L_{E S E+, j}=E_{E S E-, j} \quad(\leqslant 0) \\
L_{S E-, j}=E_{S E+, j} \quad(\geqslant 0)
\end{gathered}
$$

Inversely, the linear standard error in the propagation loss $l$ is:

$$
\begin{aligned}
l_{S E, j} & =l_{M e, j}\left(1-10^{\left(L_{S E+, j} / 20\right)}\right) \\
& =l_{M e, j}\left(10^{\left(L_{S E-j} / 20\right)}-1\right)
\end{aligned}
$$

In this way we arrive at the same value for both polarities of $l_{S E, j}$, so one calculation suffice. In the graphics we use a $\mathrm{dB}$ scale, so there the both $\mathrm{dB}$ values $L_{S E+, j}$ and $L_{S E-, j}$ are used.

\section{B. Estimation of the propagation pdf}

Next step is to estimate the propagation pdf from the measurement samples. We use the $t$-statistics for an estimation as educated in [21]. For the point estimate $\mu$ we calculate:

$$
\begin{aligned}
l_{\mu, j} & =l_{M e, j} \pm t * l_{S E, j} \text { wherein } t=0 \\
& =l_{M e, j} \\
L_{\mu, j} & =20 * \log l_{\mu, j}[\mathrm{~dB}]
\end{aligned}
$$

From the estimated standard error in the propagation loss, we want to determine the Confidence Interval, $C I$, wherein $t \in C I$. Accepting the middle $90 \%$ of the distribution, $10 \%$ is left on both trails together, so $p=0.10$, two trails. For a degrees of freedom $d f=n-1$ we look up a value for $t_{j}$ in the $t$-distribution table in [21]. The lower and upper end of the $C I$ of $l_{\mu, j}$ is now given by:

$$
\begin{gathered}
l_{\mu_{\_} \text {lower }, j}=l_{M e, j}-t_{j} * l_{S E, j} \\
l_{\mu_{-} \text {upper }, j}=l_{M e, j}+t_{j} * l_{S E, j} \\
L_{\mu_{-} \text {lower }, j}=20 * \log l_{\mu_{-} \text {lower }, j} \\
L_{\mu_{-} \text {upper }, j}=20 * \log l_{\mu_{-} \text {upper }, j}
\end{gathered}
$$

These postprocessing calculations were executed in dedicated software program written in C. Herein are the median FS values in each bin used as a basis for the propagation loss calculations, instead of the average values. 


\section{Theory and the calculations of the propagation loss}

In Appendix 2 the FS roll-off is calculated over a PEC ground. For distances $d$ in the far field region and larger than the height of the transmitter and receiver antenna the FS values are approached by:

$$
E_{P E C, d} \approx \frac{2}{d} \sqrt{\left(p_{t x} / 2\right) \cdot g_{d i p o l e} \cdot 30}=\frac{1}{d} \sqrt{p_{t x} \cdot 90}
$$

Reference [20] defines several kinds of transmission loss. However, for calculating interference risks as in relevant CISPR EMC standards often the propagation characteristics of free space is used, resulting in a $20 \mathrm{~dB}$ roll-off per decade of the distance. So for reference it is relevant to know the increase of transmission loss with distance for the free space condition.

Under far field condition, assuming an electrical dipole as transmitting antenna, we calculate the free space power density $s_{f s}$ and the field strength $e_{f s}$ as:

$$
\begin{aligned}
s_{f s} & =\frac{p_{t x} \cdot g_{d i p}}{4 \pi d^{2}} \\
e_{f s} & =\sqrt{s_{f s} \cdot Z_{0}} \approx \frac{1}{d} \sqrt{p_{t x} \cdot g_{d i p} \cdot 120 \pi / 4 \pi} \\
& \approx \frac{1}{d} \sqrt{p_{t x} \cdot 1.5 \cdot 30}
\end{aligned}
$$

The free space propagation loss ${ }^{4} L_{f s}$ we calculate from equation (20):

$$
\begin{aligned}
L_{f s} & =E_{d=1}-E_{d}[\mathrm{~dB}] \\
& =20 * \log \left(\frac{\sqrt{p_{t x} \cdot 1.5 \cdot 30}}{1}\right)-20 * \log \left(\frac{\sqrt{p_{t x} \cdot 1.5 \cdot 30}}{d}\right)(22) \\
& =20 * \log (d)
\end{aligned}
$$

From our measured FS levels we want to calculate the "Excess Propagation Loss" by comparing with the FS calculated for PEC ground and with ITU ground type "Land" [11][12] with a mediate conductivity of $3 \mathrm{mS} / \mathrm{m}$ and relative permittivity of 22. From equation (4):

$$
\begin{aligned}
& L_{\text {excess_PEC }}=E_{P E C}-E_{N, M e}[\mathrm{~dB}] \\
& L_{\text {excess_Land }}=E_{\text {Land }}-E_{N, M e}[\mathrm{~dB}]
\end{aligned}
$$

Limited to the far field ranges, we may calculate the full propagation loss $L_{f u l l}$ by:

$$
\begin{aligned}
L_{f u l l} & =L_{\text {excess_PEC }}+L_{f s} \\
& =L_{\text {excess_PEC }}+20 * \log (d)
\end{aligned}
$$

\section{STATISTICAL RESULT ANALYSIS}

Applying the foregoing calculations on the measurement results result in Figures 2, 3 and 4 for resp. 1.85, 7, 14 and 28 $\mathrm{MHz}$. In those plots the processing results are collected for the three groups of location with increasing density of habitation and for all locations totalized. Remark that the excess propagation curves related to "Land" show a transition from increasing to constant at a distance related to the wavelength. We find this transition distance back in GW field strength curves in [11], where a transition in the roll-off from $20 \mathrm{~dB} /$ dec. to $40 \mathrm{~dB} / \mathrm{dec}$. takes place. This transition is characteristic for $\mathrm{GW}$ propagation model, see [5] and [11]. We may conclude from the plots in Figures 3 - 5 that propagation in residential areas does not follow the ITU GW propagation model, but instead follow a constant slope with a constant number of $\mathrm{dBs}$ per decade of distance.

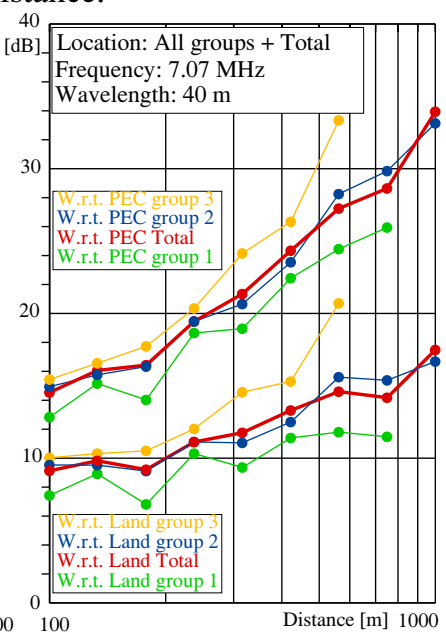

Fig. 2. Excess Propagation loss curves for 1.85 and $7 \mathrm{MHz}$.

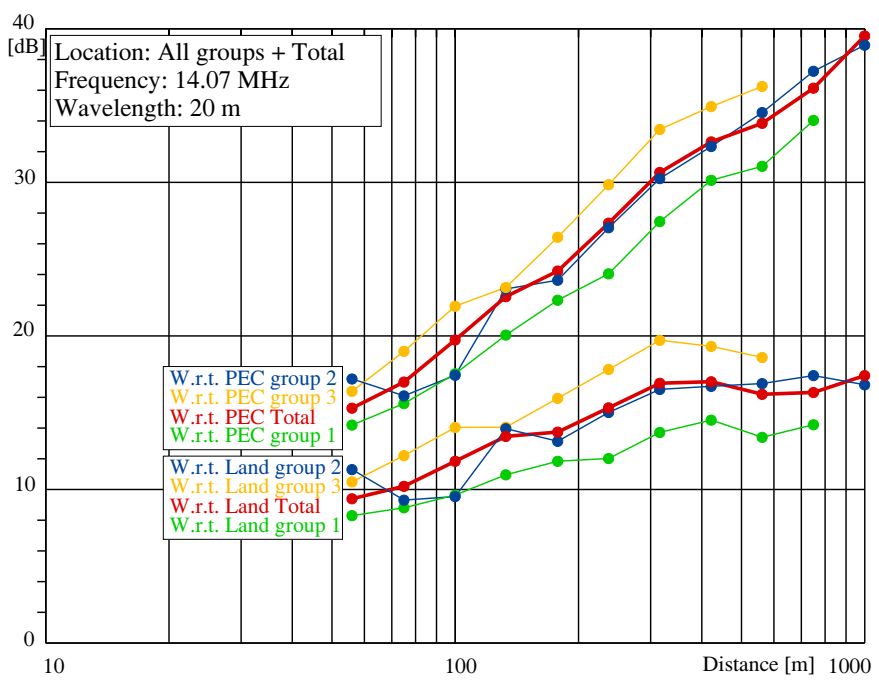

Fig. 3. Excess Propagation loss curves for $14 \mathrm{MHz}$.

\section{A. Regression analysis: Correlation to a straight line and its slope}

Inspecting the measurement results leads to the conclusion that the extra path loss above the loss according to $\mathrm{GW}$ propagation over PEC, in relation to the logarithmic value of the distance, is well approached by a straight line. For considering a correlation with a straight line we may write:

$$
L_{\text {full }}=\text { slope } * D+\alpha
$$

Now we arrive at a linear relation between $L_{f u l l}$ in $\mathrm{dB}$ and $D=20 * \log (d)$. With this relation we assume a case of interval measures, implying we may apply the Pearson correlation test, using the Pearson product-momentum

4 Here with "propagation loss" we only consider the effect of roll-off with distance of the field strength. Transmission loss as defined in [18] also takes into account the aperture of the receiving antenna, which is frequency dependent. It is defined as a ratio between the radiated power, ierp, and the received power from the lossless receiving antenna. 


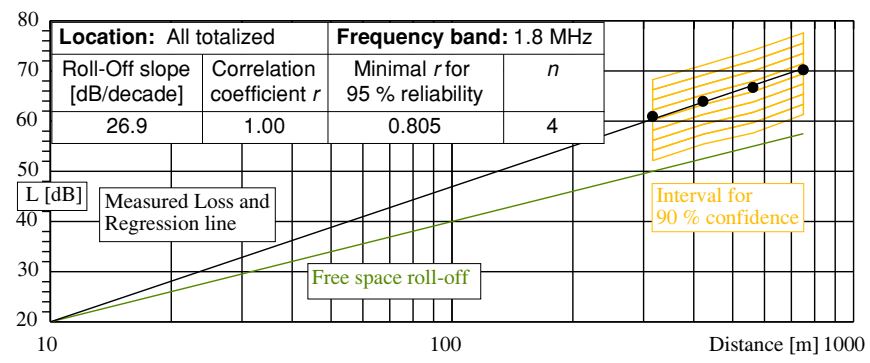

Fig. 5. Transmission loss and Roll-Off relative to $1 \mathrm{~m}$ distance at $1.8 \mathrm{MHz}$.

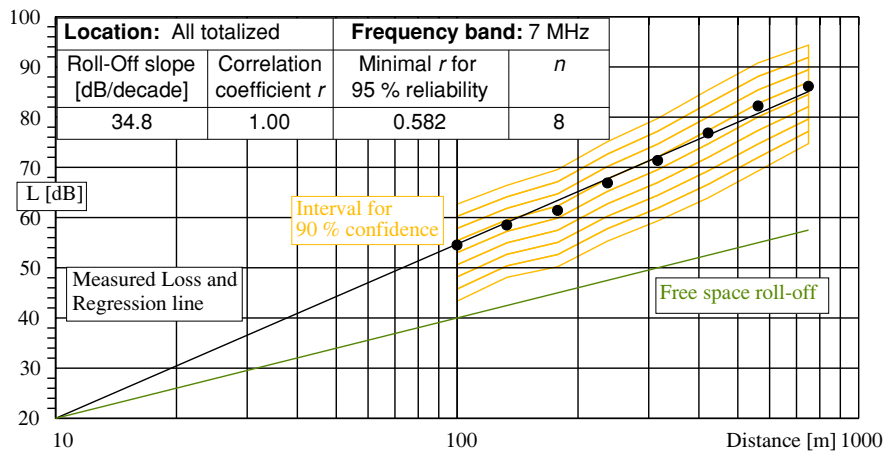

Fig. 6. Transmission loss and Roll-Off relative to $1 \mathrm{~m}$ distance at $7 \mathrm{MHz}$.

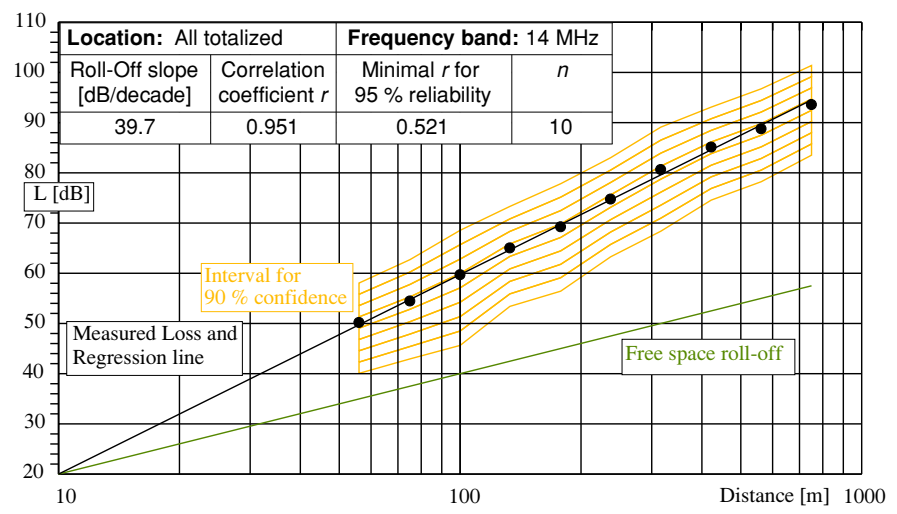

Fig. 7. Transmission loss and Roll-Off relative to $1 \mathrm{~m}$ distance at $14 \mathrm{MHz}$

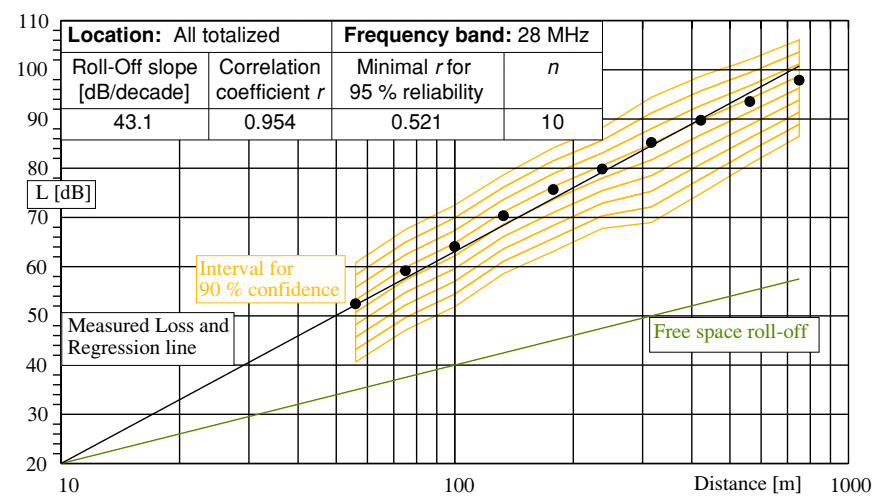

Fig. 8. Transmission loss and Roll-Off relative to $1 \mathrm{~m}$ distance at $28 \mathrm{MHz}$. 


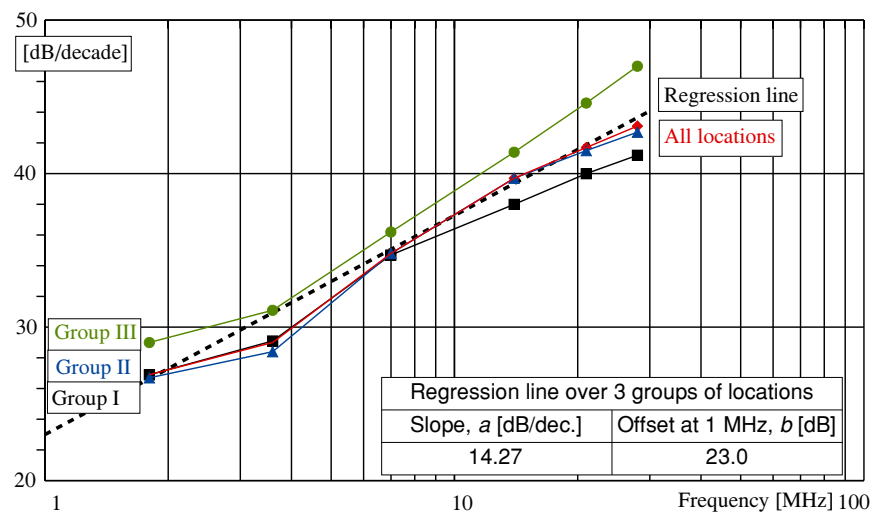

Fig. 9. Roll-Off slope as a function of frequency. Results from the three groups and all locations together.

Table II. Roll-Off slope as found by measurement in The Netherlands.

\begin{tabular}{|c|c|c|}
\hline Frequency range & Roll-Off slope: $[\mathrm{dB} /$ decade] & Slope $=\mathrm{a}^{*} \log (\mathrm{f}[\mathrm{MHz}])+\mathrm{b}$ \\
\hline $1-30 \mathrm{MHz}$ & $23-44$ & $\mathrm{a}=14.27 \quad \mathrm{~b}=23.0$ \\
\hline Low density area & $-2 \mathrm{~dB}$ & \\
\hline High density area & $+2 \mathrm{~dB}$ & \\
\hline
\end{tabular}

\section{CONCLUSIONS}

In the study of accumulation of man-made noise on MF and $\mathrm{HF}$ frequencies in urban areas propagation may play a significant role. Information from foregoing propagation studies appear to be unsatisfying, so a new experiment had to be set-up wherein a mobile field strength measurement system, in combination with a stationairy beacon transmitter, performed propagation measurements at a small scale with hundreds of measurement points at propagation distances up to 1000 meter. This method produces a high number of data and allows to run a proper statistical analysis, giving very concise results.

From these measurements at sixteen locations in The Netherlands at six frequencies from 1.8 to $28 \mathrm{MHz}$ the conclusion can be drawn that propagation in urban areas do not follow the ITU GW propagation model, but show higher propagation losses, increasing with frequency, and show a constant roll-off with distance. The slope of this roll-off is frequency dependant according a linear regression as depicted in Fig. 9 and numerically displayed in Table II. Stochastically there is a small dependency on the density of habitation, as shown in the table and visible in Fig. 9, but at individual locations the local topography is strongly relevant.

The so found statistical information about propagation loss in residential areas may be used for building an accumulation model to lay a causality between source powers, source densities, and local MMN levels. We would recommend others to do propagation experiments in other urban places, using equivalent methods, to verify and broaden our results.

\section{APPENDIX 1. VALIDATION MEASUREMENTS}

To validate this method of measurement of the propagation loss a number of validation measurement has been carried out. By definition these measurements should be done outside residential areas in open fields where the ITU GW propagation model according [11], [12], [13] should apply. As the ground in residential areas has been paved for a large part, also for the reference measurements two traject with a concrete surface were sought. The three trajects, all in the Netherlands, were:

1 A $3 \mathrm{~km}$ long, nearly straight, farmer's road near Kloosterhaar, brick road, vast open area, grown crops, only two trees halfway the road. Groundwater level: $-1.2 \mathrm{~m}$.

2 A $1000 \mathrm{~m}$ long, $25 \mathrm{~m}$ wide, runway of the general aviation airstrip near Drachten, asphalt concrete. Small airport building $150 \mathrm{~m}$ from the beacon location, business area at $150 \mathrm{~m}$ distance next to the runway at the far end.

3 A $2 \mathrm{~km}$ long, $50 \mathrm{~m}$ wide, decommissioned part of the $3 \mathrm{~km}$ runway of the military airbase Deelen, asphalt concrete, forest at $400 \mathrm{~m}$ distance at one side next to the runway. Dry sandy soil.

The measurement results are shown as normalized field strength levels next to calculated field strength levels according the ITU GW propagation model for a series of standardized types of ground, and for Perfect Electrical Conducting ground (PEC), all for an isotropic effective radiated power of $1 \mathrm{Watt}$. The measurements are performed at ten frequencies in the range from 1.8 to $28 \mathrm{MHz}$. Because of limited space we will show the plots of three frequencies here, showing the three locations together.

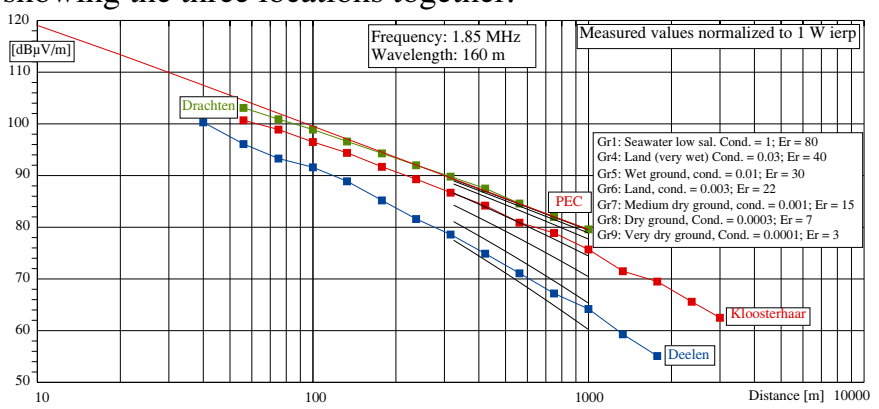

Fig. A1.1. Field strength roll-off with distance, measured and compared with ITU GW propagation model at $1.85 \mathrm{MHz}$.

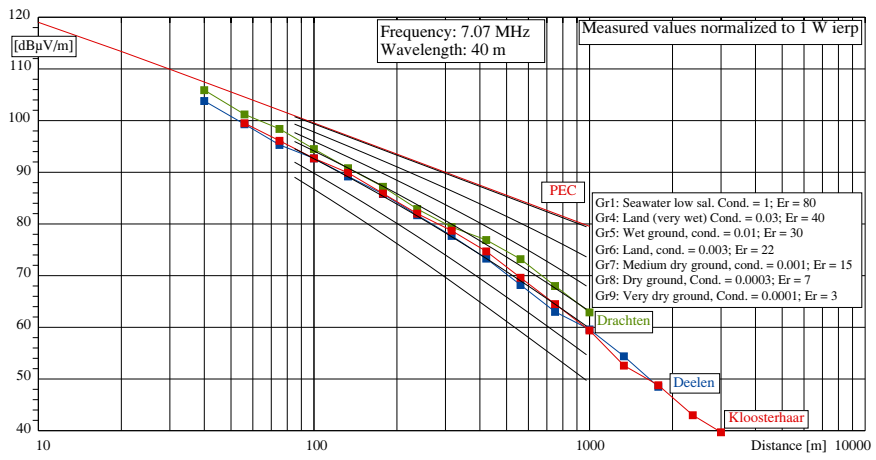

Fig. A1.2. Field strength roll-off with distance, measured and compared with ITU GW propagation model at 7.07 $\mathrm{MHz}$.

A few observations we will mention here. In the measurements at Kloosterhaar we see a good propagation for lower frequencies and bad propagation for the higher frequencies. We explain that by the influence of the groundwater causing an enhanced conductivity deeper in the ground, having influence at the longer wavelength. At the higher frequencies the extra losses caused by vegetation is having an effect, especially the effect of the 2 meter high crops in the distance range of 200 to $700 \mathrm{~m}$ is visible. At Deelen the underground is sandy and dry, so bad propagation for low frequencies, but for the high frequencies the effect of 
the runway pavement appear to be relevant. In the measurement on the runway of Drachten the conducting effect of buried cables from decommissioned runway lighting is showing up on the lower frequencies. We conclude that these measurements show results being well inside the range we might expect, and so validate the measurement system and method.

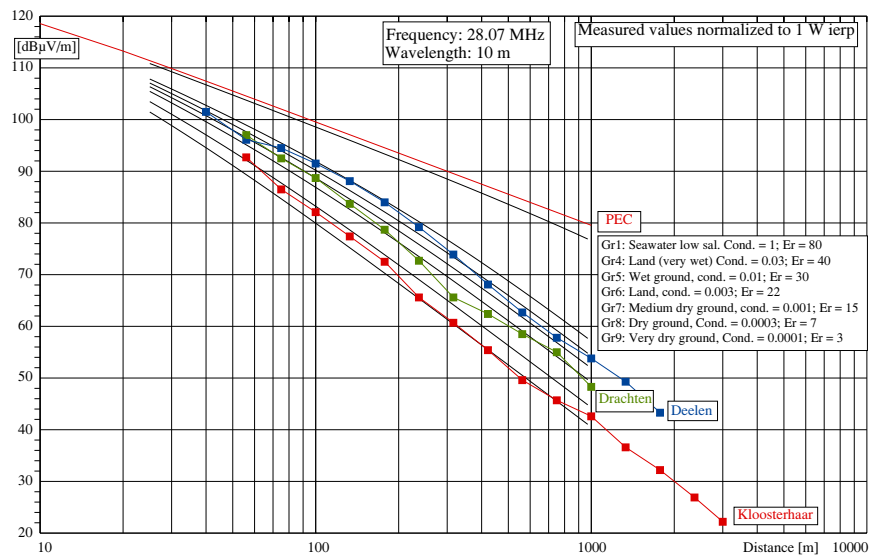

Fig. A1.3. Field strength roll-off with distance, measured and compared with ITU GW propagation model at $28.07 \mathrm{MHz}$.

\section{APPENDIX 2: CALCULATION OF FIELD STRENGTH ABOVE PERFECT ELECTRICAL CONDUCTING GROUND}

When the antenna is elevated, for example as an asymmetric dipole (Fig. A2.1), we must take into account the direct ray from transmitting antenna to the measurement antenna, and the reflected ray against ground. The reflected wave is thought to be radiated by a mirrored antenna under the ground. A simplified way, using PEC, is as follows.

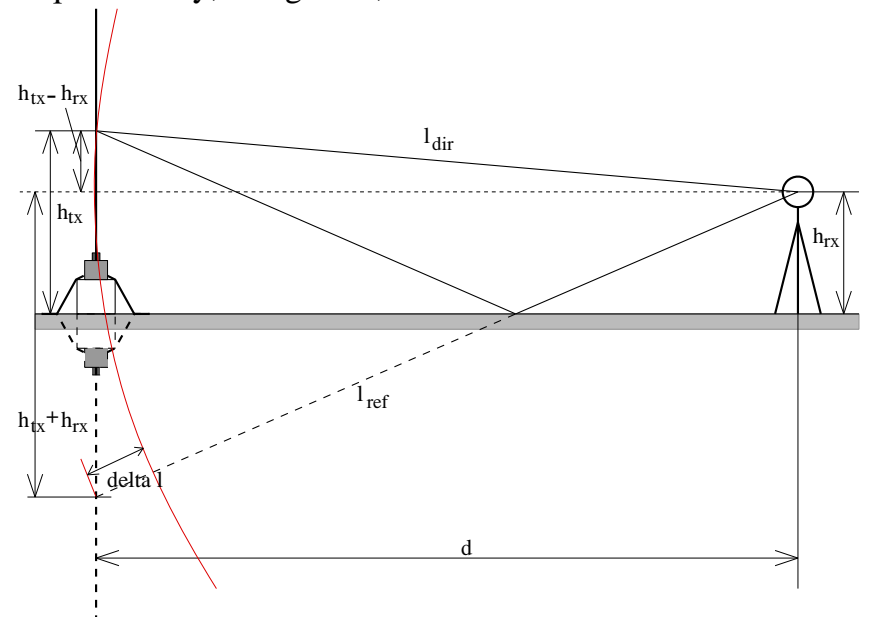

Fig. A2.1. Model of radiation from an asymetric dipole.

When we calculate the field strength using the mirror transmit antenna method, we must divide the radiated power over the two antenna parts. Further, we must apply the gain of a small electrical dipole, $g_{d i p}=1.5$, for each antenna part, [22]. Direct wave:

$$
s_{d i r}=\frac{p_{t x}}{2} \cdot \frac{g_{d i p}}{4 \pi l_{d i r}^{2}}
$$

$$
\begin{aligned}
& e_{d i r}=\sqrt{s_{d i r} \cdot Z_{0}} \approx \frac{1}{l_{d i r}} \sqrt{p_{t x} \cdot g_{d i p} \cdot 15} \\
& l_{d i r}=\sqrt{d^{2}+\left(h_{t x}-h_{r x}\right)^{2}}
\end{aligned}
$$

Ground reflected wave:

$$
\begin{aligned}
& s_{r e f}=\frac{p_{t x}}{2} \cdot \frac{g_{d i p}}{4 \pi l_{r e f}^{2}} \\
& e_{r e f}=\sqrt{s_{r e f} \cdot Z_{0}} \approx \frac{1}{l_{r e f}} \sqrt{p_{t x} \cdot g_{d i p} \cdot 15} \\
& l_{\text {ref }}=\sqrt{d^{2}+\left(h_{t x}+h_{r x}\right)^{2}}
\end{aligned}
$$

The total wave is the vectorial sum of both path. Let

$$
\begin{aligned}
& \operatorname{Re}\left(e_{d i r}\right)=\left|e_{d i r}\right| \\
& \operatorname{Im}\left(e_{d i r}\right)=0 \\
& \operatorname{Re}\left(e_{r e f}\right)=\left|e_{r e f}\right| \cdot \cos \phi \\
& \operatorname{Im}\left(e_{r e f}\right)=\left|e_{r e f}\right| \cdot \sin \phi
\end{aligned}
$$

Angle $\phi$ is caused by the delay by the extra path length $\Delta l=l_{\text {ref }}-l_{\text {dir }}$

$$
\begin{aligned}
\phi & =\frac{\Delta l}{\lambda} 2 \pi \quad(\Delta l \leqslant \lambda) \\
\operatorname{Re}\left(e_{t o t}\right) & =\operatorname{Re}\left(e_{d i r}\right)+\operatorname{Re}\left(e_{r e f}\right) \\
& =\left|e_{d i r}\right|+\left|e_{r e f}\right| \cdot \cos \phi \\
\operatorname{Im}\left(e_{t o t}\right) & =\operatorname{Im}\left(e_{d i r}\right)+\operatorname{Im}\left(e_{r e f}\right) \\
& =\left|e_{r e f}\right| \cdot \sin \phi \\
\left|e_{t o t}\right| & =\sqrt{\left(\operatorname{Re}\left(e_{t o t}\right)\right)^{2}+\left(\operatorname{Im}\left(e_{t o t}\right)\right)^{2}} \\
& =\sqrt{\left(\left|e_{d i r}\right|+\left|e_{r e f}\right| \cdot \cos \phi\right)^{2}+\left(\left|e_{r e f}\right| \cdot \sin \phi\right)^{2}}( \\
& =\sqrt{\left|e_{d i r}\right|^{2}+\left|e_{r e f}\right|^{2}+2 \cdot\left|e_{d i r}\right| \cdot\left|e_{r e f}\right| \cdot \cos \phi}
\end{aligned}
$$

At distances larger than the transmitter and receiver antenna heights, so for $l_{d i r}=l_{\text {ref }}=d$ and $\phi=0$ :

$$
\begin{aligned}
e_{d i r} & =e_{r e f}=e=\frac{1}{d} \sqrt{p_{t x} \cdot g_{d i p} \cdot 15} \\
\left|e_{t o t}\right| & =\sqrt{|e|^{2}+|e|^{2}+2 \cdot|e| \cdot|e|}=2 \cdot e \\
& =\frac{2}{d} \sqrt{p_{t x} \cdot g_{d i p} \cdot 15} \\
& =\frac{1}{d} \sqrt{p_{t x} \cdot 90}
\end{aligned}
$$

\section{APPENDIX 3. COMPENSATION FOR MEASURING IN THE NEAR FIELD}

Reference [23], chapter 38, give a good description of the near fields, electric and magnetic, of a small oscillating dipole. From there it is not difficult to derive equations that describe the distance function in the main radiation direction for the Eand H-field: 


$$
\begin{aligned}
|E| & =\frac{[p]}{4 \pi \varepsilon_{0} \lambda^{2} r} \sqrt{\left(\frac{\lambda^{2}}{r^{2}}-1\right)^{2}+\left(\frac{\lambda}{r}\right)^{2}} \\
& =\frac{[p]}{4 \pi \varepsilon_{0} \lambda^{2} r} \cdot k_{E} \\
k_{E} & =\sqrt{\left(\frac{\lambda^{2}}{r^{2}}-1\right)^{2}+\left(\frac{\lambda}{r}\right)^{2}} \\
|H| & =\frac{c[p]}{4 \pi \lambda^{2}} \sqrt{(-1)^{2}+\left(\frac{\lambda}{r}\right)^{2}} \\
& =\frac{c[p]}{4 \pi \lambda^{2}} \cdot k_{H} \\
k_{H} & =\sqrt{1+\left(\frac{\lambda}{r}\right)^{2}}
\end{aligned}
$$

Wherein $k_{E}$ and $k_{H}$ represents the correction factors in the near field for the electric, resp. the magnetic field component. In the far field with $r \gg \lambda: k_{E}=1$ and $k_{H}=1$. Herein the radian wavelength $\lambda$ is defined by:

$$
\lambda=\frac{\lambda}{2 \pi}
$$

\section{REFERENCES}

[1] T.W.H. Fockens, A.P.M. Zwamborn, and F. Leferink, "Measurements of the Man-made Noise Floor on HF in The Netherlands", IEEE Transactions on EMC, vol. 61, no. 2, April 2019 DOI: 10.1109/ TEMC.2018.2830512.

[2] T.W.H. Fockens and F. Leferink, "Correlation Between Measured ManMade Noise Levels and the Density of Habitation", IEEE Transactions on $E M C$, vol. 62, no. 6, December 2020, DOI: 10.1109/ TEMC.2020.3001979.

[3] J. Zenneck, "Propagation of Plane EM Waves Along a Plane Conducting Surface," Ann. Phys. (Leipzig), 28, 1907, pp. 846-866.

[4] A.N. Sommerfeld, "Propagation of Waves in Wireless Telegraphy," Ann. Phys. (Leipzig), 28, 1909, pp. 665-737.

[5] K.A. Norton, "The Propagation of Radio Waves over the Surface of the Earth and in the Upper Atmosphere, Part I", Proceeding of the IRE, vol. 24, no. 10,1936 , pp. 1367-1387.

[6] K.A. Norton, "The Propagation of Radio Waves over the Surface of the Earth and in the Upper Atmosphere, Part II", Proceeding of the IRE, vol. 25, no. 9,September1937, pp. 1203-1236.

[7] K.A. Norton, "The Physical Reality of Space and Surface Waves in the
Radiation field of Radio Antennas", Proceeding of the IRE, vol. 25, no. 9, September1937, pp. 1192-1202.

[8] J.R. Wait, "The Ancient and Modern History of EM Ground-Wave Propagation", IEEE Antennas and Propagation Magazine, vol. 40, no. 5, October 1998, pp. 7-24.

[9] R.E. Collin, "Hertzian Dipole Radiating Over a Lossy Earth or Sea: Some Early and Late 20th-Century Controversies", IEEE Antennas and Propagation Magazine, vol. 46, no. 2, April 2004, pp. 64-79.

[10] K.A. Norton, "The Calculation of Ground-Wave Field Intensity Over a Finitely Conducting Spherical Earth", Proceeding of the IRE, vol. 29, December 1941, pp. 623-639.

[11] Ground-wave propagation curves for frequencies between $10 \mathrm{kHz}$ and 30 $M H z$. Rec. ITU-R P.368-9 (2007).

[12] Handbook on Ground Wave Propagation, 2014, ITU.

[13] GRWAVE. Ground wave simulating tool from ITU-R.

[14] Yoanne Corre, Yves Lostanlen, "Three-Dimensional Urban EM Wave Propagation Model for Radio Network Planning and Optimization Over Large Areas", IEEE Transactions on Vehicular Technology, vol. 58, no. 7, pp. 3112 - 3123, September 2009, DOI: 10.1109/TVT.2009.2016973.

[15] Vittorio Degli-Esposti, "A Diffuse Scattering Model for Urban Propagation Prediction", IEEE Transaction on Antennas and Propagation, vol. 49, no. 7, July 2001, pp. 111- 1113.

[16] J.H. Causebrook, "Medium-Wave propagation in built-up areas", Proceedings IEE, vol. 125, no. 9, September 1978, pp. 804-808.

[17] M.P.C. Almeida, e.a. "Medium wave DRM field trails in Brazil - Some daytime and nighttime results in urban environment", Measurement, Elsevier, vol. 45, year 2012, pp. 2237-2245.

[18] M. J. Packer, R.I. Desourdis, "Rural and Urban Groundwave propagation in a desert environment", Proceedings of MILCOM '93 - IEEE Military Communications Conference, 1993, vol. 2, pp. 634-638.

[19] L. Lichun, "A New MF and HF Ground-Wave Model for Urban Areas", IEEE Antennas and Propagation Magazine, vol. 42, no. 1, February 2000, pp. 21-33.

[20] The concept of transmission loss for radio links. Rec. ITU-R P.341-7 (2019)..

[21] Statistics for the Behavioral sciences, Fr.J. Gravettes and L.B. Wallnau., 7th edition, Thomson - Wadsworth ISBN-13: 978-0-495-09521-7.

[22] Antennas for All Applications. John D. Kraus and Ronald J. Marhefka, MacGraw-Hill Higher Education, Edition 3, 2002. ISBN 0-07-112240-0.

[23] Electromagnetic Fields and Waves, Paul Lorrain, Dale P. Corson, and François Lorrain, 3rd edition, W.H. Freeman and Company, 1988. ISBN: 0-716-71823-5. 